\title{
BeppoSAX Low-Energy Concentrator Spectrometer background subtraction techniques
}

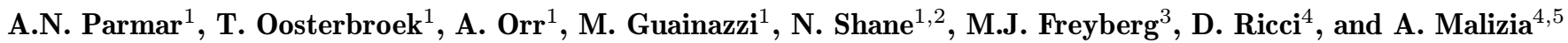 \\ 1 Astrophysics Division, Space Science Department of ESA, ESTEC, Postbus 299, 2200 AG Noordwijk, The Netherlands \\ 2 Pembroke College, University of Oxford, St Aldates, Oxford, OX1 1DW, UK \\ 3 Max-Planck-Institut für Extraterrestrische Physik, D-85740 Garching bei München, Germany \\ ${ }^{4}$ BeppoSAX Science Data Center, Nuova Telespazio, via Corcolle 19, I-00131 Roma, Italy \\ 5 Department of Physics and Astronomy, Southampton University, SO17 1BJ, UK
}

Received November 17, 1998; accepted February 2, 1999

\begin{abstract}
We present 3 methods for the subtraction of non-cosmic and unresolved cosmic backgrounds observed by the Low-Energy Concentrator Spectrometer (LECS) on-board BeppoSAX. Removal of these backgrounds allows a more accurate modeling of the spectral data from point and small-scale extended sources. At high $\left(>|25|^{\circ}\right)$ galactic latitudes, subtraction using a standard background spectrum works well. At low galactic latitudes, or in complex regions of the X-ray sky, two alternative methods are presented. The first uses counts obtained from two semi-annuli near the outside of the LECS field of view to estimate the background at the source location. The second method uses ROSAT Position Sensitive Proportional Counter (PSPC) all-sky survey data to estimate the LECS background spectrum for a given pointing position. A comparison of the results from these methods provides an estimate of the systematic uncertainties. For high galactic latitude fields, all 3 methods give $3 \sigma$ confidence uncertainties of $<0.910^{-3}$ count $\mathrm{s}^{-1}$ $(0.1-10 \mathrm{keV})$, or $<1.510^{-3}$ count $\mathrm{s}^{-1}(0.1-2 \mathrm{keV})$. These correspond to $0.1-2.0 \mathrm{keV}$ fluxes of $0.7-1.8$ and $0.5-1.110^{-13} \mathrm{erg} \mathrm{cm}^{-2} \mathrm{~s}^{-1}$ for a power-law spectrum with a photon index of 2 and photoelectric absorption of $310^{20}$ and $310^{21}$ atom $\mathrm{cm}^{-2}$, respectively. At low galactic latitudes, or in complex regions of the X-ray sky, the uncertainties are a factor $\sim 2.5$ higher.
\end{abstract}

Key words: instrumentation: detectors - methods: data analysis — diffuse radiation - X-rays: general

Send offprint requests to: A.N. Parmar (aparmar@astro.estec. esa.nl)

\section{Introduction}

The Low-Energy Concentrator Spectrometer (LECS) (Parmar et al. 1997b) is an imaging gas scintillation proportional counter on-board the Italian-Dutch BeppoSAX X-ray astronomy mission. BeppoSAX (Boella et al. 1997a) was launched into a $600 \mathrm{~km} 3.9$ inclination orbit on 1996 April 30. The LECS is one of four coaligned narrow field instruments (NFIs). The other NFIs are the imaging Medium-Energy Concentrator Spectrometer (MECS; $1.3-10 \mathrm{keV}$; Boella et al. 1997b), the High Pressure Gas Scintillation Proportional Counter (HPGSPC; $5-120 \mathrm{keV}$; Mazo et al. 1997) and the Phoswich Detection System (PDS; $15-300 \mathrm{keV;} \mathrm{Frontera}$ et al. 1997). In addition, the payload includes two wide field cameras (WFC; $2-30 \mathrm{keV}$; Jager et al. 1997) which observe in directions perpendicular to the NFI. The MECS background is discussed in Chiappetti et al. (1998).

Accurate background subtraction is important for virtually all X-ray observations and especially for studies of extended and diffuse objects. In this paper we discuss background subtraction techniques appropriate to the LECS. Background subtraction is complicated in the LECS because (1) the wings of the mirror point spread function distribute a significant fraction of hard X-rays over the entire field of view (FOV), (2) the broad detector response spreads a fraction of low-energy X-rays over the entire FOV, (3) the off-axis response of the mirrors is complicated and single scattered X-rays from outside the direct FOV can reach the focal plane (Conti et al. 1994), and (4) at low-energies the X-ray sky has a great deal of spatial structure (see e.g., Snowden et al. 1995, 1997). This spatial structure has a complex energy dependence. Below $0.28 \mathrm{keV}$ there is a galactic plane-to-pole variation of a factor $\sim 3$, while at energies between 0.5 and $2.0 \mathrm{keV}$ 
the North Polar Spur and the galactic bulge dominate. For energies $>0.5 \mathrm{keV}$ and $50^{\circ}<l_{\mathrm{II}}<290^{\circ}$ the X-ray sky is relatively smooth with few discrete features visible.

\section{The LECS}

The method of operation of the LECS is similar to that of conventional gas scintillation proportional counters. An $\mathrm{X}$-ray that passes through the ultra-thin $(1.25 \mu \mathrm{m})$ entrance window and is absorbed in the $11.4 \mathrm{~cm}$ diameter Xe filled gas cell liberates a cloud of electrons. A uniform electric field between the entrance window, kept at $-20 \mathrm{kV}$, and a grounded grid causes scintillation as the electrons travel towards the grid. The UV light from these scintillations is detected by a multi-anode photo-multiplier tube (PMT). The LECS is also sensitive to cosmic rays since these can ionize the counter gas and leave tracks through the cell. The $2 \mathrm{~cm}$ diameter gas cell entrance window is supported by a tungsten strongback and fine grid. The strongback consists of a square grid of tungsten bars with a separation of $4^{\prime}$. Each strongback square is further divided into 8 by 8 pixels by a fine grid. The overall effect of the strongback and fine grid is to reduce the $\mathrm{X}$-ray transmission by between $20-40 \%$, depending on position within the FOV. The LECS includes two ${ }^{55} \mathrm{Fe}$ radioactive sources which constantly illuminate regions of the detector outside the sky FOV to provide monitoring of the instrument performance (see Fig. 1).

The LECS mirror system consists of 30 nested, Au coated mirrors with a double cone approximation to the Wolter I geometry. The mirror focal length is $185 \mathrm{~cm}$ and the geometric area $124 \mathrm{~cm}^{2}$ (Conti 1994, 1996). The offaxis behavior of the mirrors is complicated. Conti et al. (1994) demonstrate that X-rays within a cone of solid angle $2^{\circ}$ can reach the focal plane after reflection off only one of the mirror surfaces. As part of the BeppoSAX Science Verification Phase, observations were performed with the Crab Nebula just outside the LECS FOV. These show that at an offset of $60^{\prime}$, the Crab Nebula is visible as an extended emission region offset in the direction of the source, with an intensity $(5.2 \pm 1.8) 10^{-4}$ of that on-axis. The spectrum of the offset emission (a power-law with a photon index, $\alpha$, of $2.2 \pm 0.2$ ) is consistent with that recorded from the Crab Nebula on-axis $(\alpha=2.1)$.

The LECS is only operated during satellite night time. Although this reduces the observing efficiency considerably, it means that that any contribution to the background from scattered solar X-rays, e.g., as seen by the ROSAT Position Sensitive Proportional Counter (PSPC) when the Sun-Earth-satellite angle is $<120^{\circ}$ (e.g., Snowden \& Freyberg 1993), is negligible. The BeppoSAX observing schedule is designed to optimize the LECS observing efficiency. This means that, in general, only time constrained or Target of Opportunity observations include long intervals of dark Earth pointing.

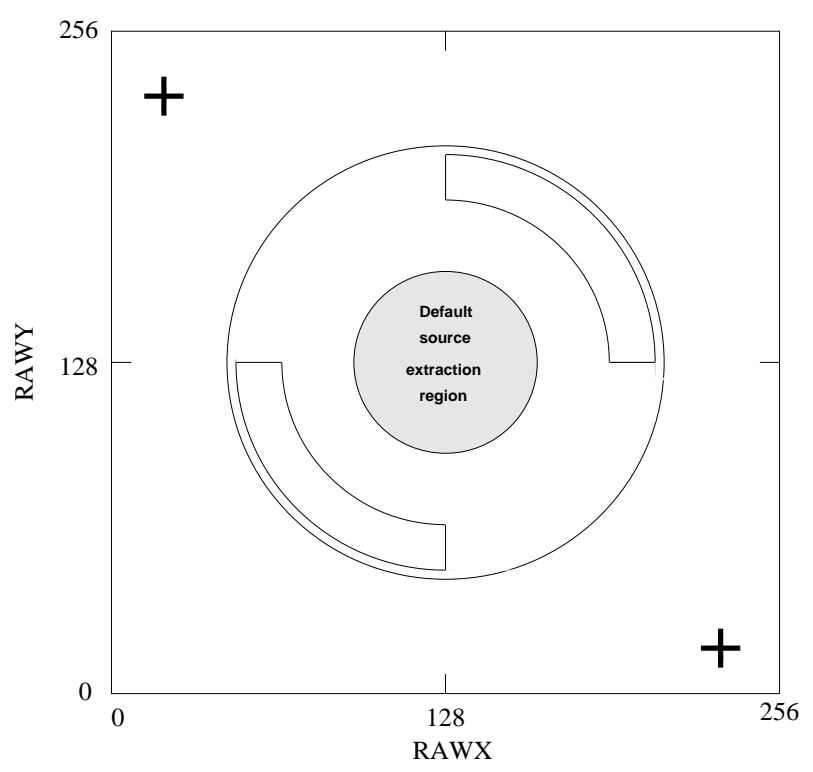

Fig. 1. The LECS FOV drawn to scale. The outer circle indicates the $37^{\prime}$ diameter sky FOV which contains the standard $16^{\prime}$ diameter source extraction region (shaded) and the two background semi-annuli. The positions of the two ${ }^{55} \mathrm{Fe}$ radioactive sources are shown as crosses

The LECS is not operated when the satellite passes close to the South Atlantic Anomaly (SAA). Due to the low BeppoSAX orbital inclination SAA passages result in between 5 and $12 \mathrm{~min}$ of data being lost per 96 min satellite orbit. In order to maximize the observing efficiency, many BeppoSAX NFI observations are made with a Sun-satellite-target angle close to $120^{\circ}$, which is as far from the solar direction as it is possible to operate.

The LECS energy resolution is $32 \%$ full-width halfmaximum (FWHM) at $0.28 \mathrm{keV}$ and $8.8 \%$ FWHM at $6 \mathrm{keV}$. The FOV is circular with a diameter of $37^{\prime}$. The position resolution corresponds to $90 \%$ encircled energy within a radius of 2.5 at $1.5 \mathrm{keV}$. This is a factor $\sim 4$ worse than that of the PSPC and comparable to that of the ASCA Gas Imaging Spectrometer (GIS). Figure 2 shows the on-axis LECS effective area. A key scientific goal of the LECS is to study sources in the energy band below the instrument's C edge at $0.28 \mathrm{keV}$. This typically means that the absorption to a source must be $\lesssim 310^{21}$ atom $\mathrm{cm}^{-2}$. The prime advantage of the LECS, compared to previous high throughput X-ray detectors, is its good low-energy spectral resolution and its low background afforded by the imaging characteristics. The $0.1-2.0 \mathrm{keV}$ energy resolution is a factor $\sim 2.4$ better that that of the PSPC, while the effective area is between a factor $\sim 20$ and 5 lower at $0.28 \mathrm{keV}$ and $1.5 \mathrm{keV}$, respectively. The energy resolution is similar to that of the GIS in the overlapping energy range, and comparable to that of the ASCA Solid-state Imaging Spectrometer at energies of $\sim 0.5 \mathrm{keV}$. 


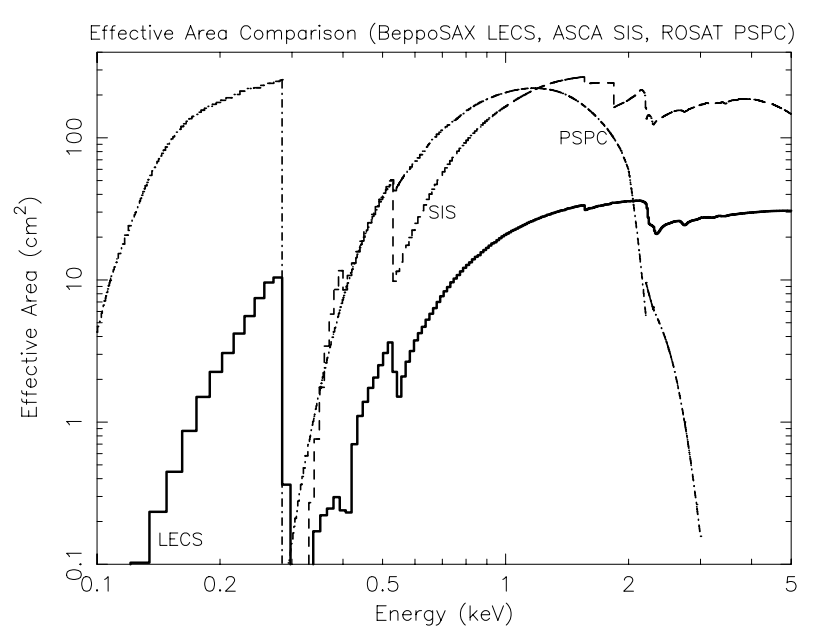

Fig. 2. A comparison of the LECS (solid line), ASCA SIS (dashed line) and ROSAT PSPC (Dashed-dotted line) effective areas in the energy range $0.1-5.0 \mathrm{keV}$

\section{Data selection and processing}

In order to study the properties of the LECS background, data were extracted from 350 BeppoSAX Final Observation Tapes, corresponding to 170 individual observations. These data were processed using the standard LECS processing pipeline described below and cleaned event lists for the intervals when the instrument was observing both the dark Earth and the sky in the nominal operating configuration extracted. Observations with $<100$ counts were excluded. This left total sky and dark Earth exposures of $2847 \mathrm{ks}$ and $498.6 \mathrm{ks}$, respectively.

Table 1 gives the contents of the LECS telemetry for each detected event. The standard LECS data processing package (SAXLEDAS 1.8.0) performs the following steps to produce cleaned, linearized event lists:

1. Conversion of RAWX and RAWY into linear coordinates referred to as DETX and DETY using the algorithm given in Parmar et al. (1997b).

2. Determination of the overall instrument gain (which is temperature dependent), using the mean energy of the two calibration sources as a reference.

3. Conversion of Pulse height channels to Pulse Invariant (PI) channels which have a fixed relation to energy. This conversion takes into account the overall gain and a FOV position dependent gain correction.

4. Selection of intervals with nominal instrument settings (such as high voltage settings), and separation into events that are $>4^{\circ}$ from the Earth's limb (taken to be good on-target intervals), and those where the dark Earth occults the FOV.

5. Removal of background events using the VETO and BL signals. The VETO signal is the ratio between the light measured by the central PMT anode and the sum of that measured by the surrounding anodes. It therefore has a high value for on-axis X-ray sources and
Table 1. LECS telemetry contents

\begin{tabular}{lll}
\hline Parameter & Range & Comment \\
\hline RAWX & $0-255$ & Event $X$ coordinate \\
RAWY & $0-255$ & Event $Y$ coordinate \\
TIME & $\ldots$ & Event Time (units of $16 \mu \mathrm{s})$ \\
PHA & $0-1023$ & Event pulse height (energy) \\
VETO & $0-255$ & Event light distribution \\
BL & $0-255$ & Event burstlength \\
\hline
\end{tabular}

lower values for events occurring further off-axis. In SAXLEDAS 1.8 .0 a sliding cell VETO window is applied to the data. The BL signal is a measure of the duration of the light flash produced following absorption in the gas cell. It depends on the penetration depth of an event into the cell, which is energy dependent. In SAXLEDAS 1.8.0 events are selected if their PI and BL values are within a set of 62 pre-defined boxes.

The standard LECS data analysis technique is to extract counts within a radius of $8^{\prime}$ (corresponding to 35 RAW pixels; see Fig. 1) from the source centroid using the cleaned event lists produced by SAXLEDAS. This large radius ensures that $95 \%$ of the $0.28 \mathrm{keV}$ X-rays are included in the analysis. For absorbed, or very faint sources, extraction radii of $4^{\prime}$ or $6^{\prime}$ may be appropriate. The BeppoSAX pointing accuracy is such that most target sources are located within $\sim 2^{\prime}$ of the nominal position of RAWX, RAWY = 131, 124.

\section{Standard background}

The usual method for LECS background subtraction is to subtract the counts obtained in the same detector region using a standard background exposure. This consists of the sum of the blank field exposures listed in Table 2. The LECS data for these fields were processed using SAXLEDAS 1.8.0. No point sources are present in the individual fields with $0.1-2.0 \mathrm{keV}$ fluxes $>1.710^{-13} \mathrm{erg} \mathrm{s}^{-1}$, or $2-10 \mathrm{keV}$ fluxes of $>9.710^{-14} \mathrm{erg} \mathrm{s}^{-1}$. Searches were also made of X-ray catalogs such as the ROSAT Bright Source Catalog (Voges et al. 1996) to check for the presence of contaminating point sources in, and a couple of degrees outside the FOV. Note that the Gal cent- 2 and -3 fields include the complex region of sky around the Draco nebula (e.g., Kerp 1994; Moritz et al. 1998). A $22.1 \mathrm{ks}$ BeppoSAX background exposure on 1996 August 11 at $\mathrm{RA}=14^{\mathrm{h}} 42^{\mathrm{m}} 27^{\mathrm{s}} .1$, Dec $=+19^{\circ} 44^{\prime} 10^{\prime \prime}$ was not included in the standard background since it is in the direction of the North Polar Spur. Examination of the LECS spectrum showed that it has significantly more low-energy flux than the fields listed in Table 2. The remaining exposure time is $558.6 \mathrm{ks}$.

The standard background $0.1-10.0 \mathrm{keV}$ count rate in the central $8^{\prime}$ radius source extraction region is 

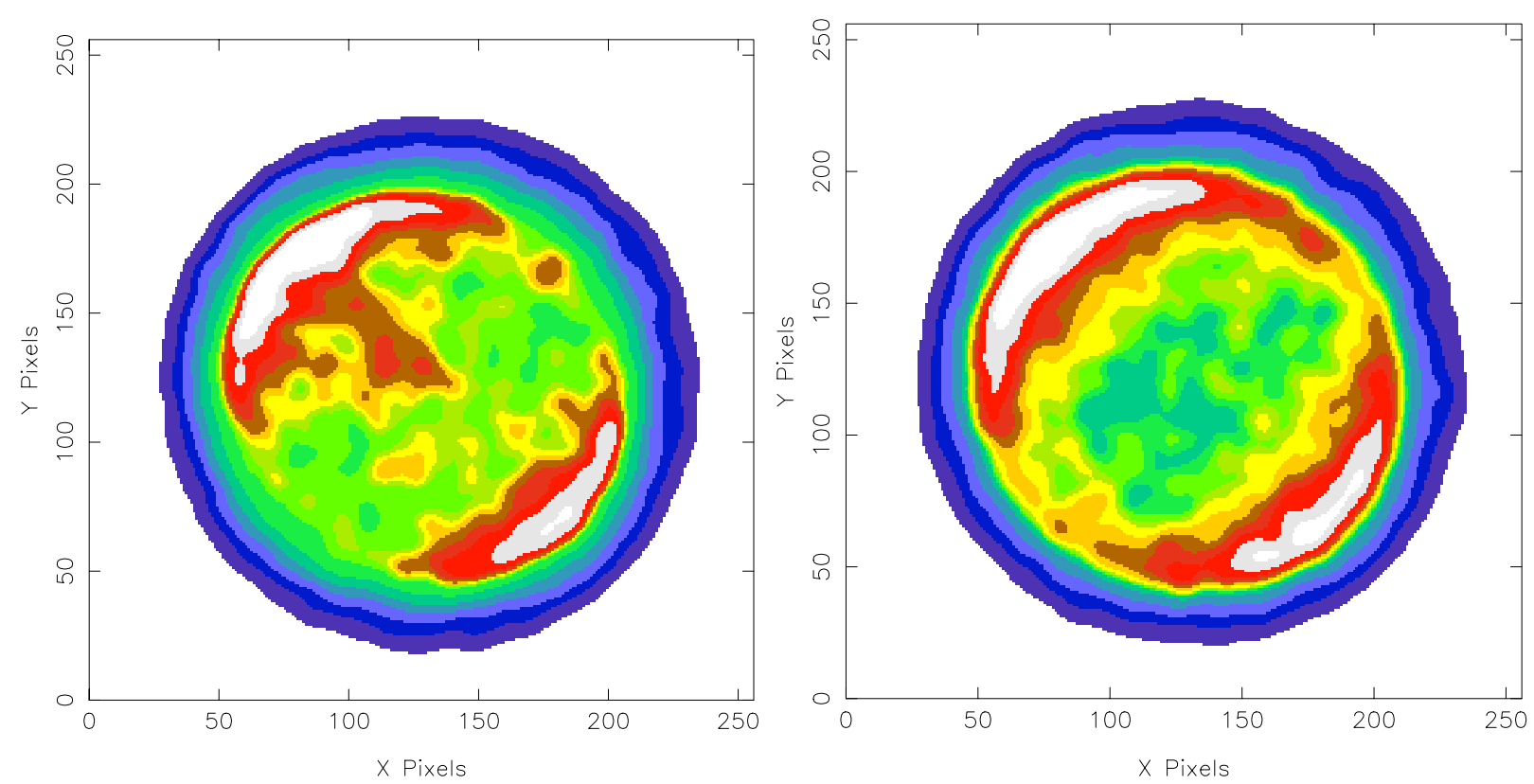

Fig. 3. The dependence of the LECS standard background (left panel) and NXB (right panel) on RAWX, RAWY position within the FOV. Gaussian smoothing filters with a $\sigma$ of 4 pixels have been applied

$1.010^{-5} \mathrm{~s}^{-1} \mathrm{keV}^{-1} \operatorname{arcmin}^{-2}$. The dependence of the standard background count rate on position within the FOV is shown in the left panel of Fig. 3. This image has been smoothed using a Gaussian filter with a $\sigma$ of 4 RAW pixels. The difference in intensity between the most and least intense regions is a factor 1.8. The $\mathrm{X}$-ray background appears brighter close to the center of the image due primarily to mirror vignetting. Note that the LECS mirror axis is offset by $3^{\prime}$ from the center of the FOV due to an alignment error during integration. The two bright arcs are located radially inwards from the ${ }^{55} \mathrm{Fe}$ calibration sources and result from $5.9 \mathrm{keV}$ characteristic X-rays that are absorbed some distance from their origin.

Figure 4 illustrates the overall shape of the standard background spectrum obtained in the central $8^{\prime}$ of the FOV. The Non X-ray background (NXB) obtained from the same region of the detector is also shown (see Sect. 5). The difference between the two is the contribution of the cosmic X-ray background (CXB), spectral fits to which are presented in a companion paper (Parmar et al. 1999).

\section{Non X-ray background (NXB)}

A total of $498.6 \mathrm{ks}$ of NXB data was accumulated using LECS dark Earth pointings. The dependence of the $0.1-10 \mathrm{keV}$ count rate, $C_{\mathrm{T}},\left(\mathrm{s}^{-1}\right)$ on time is shown in Fig. 5, where each data point is averaged over the entire FOV. The interval without data points results from successive gyro failures on BeppoSAX, which led to a 3 month observing hiatus. A gradual reduction in the counting rate by $\sim 15 \%$ over an interval of $\sim 2$ years is evident. This may be modeled as $C_{\mathrm{T}}=0.1069-2.210^{-5} \Delta T$, where $\Delta T$ is the number of days since launch. Alternatively, an exponential fit indicates a decay constant of 11.8 years. The same trend is visible in data extracted from the central $8^{\prime}$ radius and background semi-annuli regions (see Sect. 6). The intensity of the primary $\left(5.89 \mathrm{keV} \mathrm{Mn} \mathrm{K}_{\alpha}\right.$ and $6.49 \mathrm{keV} \mathrm{Mn} \mathrm{K}_{\beta}$ ) radiation from the ${ }^{55} \mathrm{Fe}$ calibration sources decays with a much shorter half-life of 2.73 years. However, the calibration sources contain small amounts of other radioactive materials, the decay of which could contribute to the decrease in NXB intensity. Another factor may be the decreasing particle background in low-Earth orbit as the next solar maximum is approached, due to the varying height of the Earth's atmosphere. Such an effect is evident in studies of the ROSAT High Resolution Imager NXB (Snowden 1998). There is little evidence in the LECS data for "Long Term Enhancements" or LTEs as seen by the PSPC (Snowden et al. 1995). These count rate increases are strongest below $0.28 \mathrm{keV}$ and are approximately uniform over an orbit. They last between $15-30$ ROSAT orbits and have peak intensities comparable to the low-energy CXB.

In the central $8^{\prime}$ radius extraction region the $0.1-$ $10 \mathrm{keV} \mathrm{NXB}$ count rate is $5.210^{-6} \mathrm{~s}^{-1} \mathrm{keV}^{-1} \operatorname{arcmin}^{-2}$. The dependence of the NXB on position within the FOV is shown in the right panel of Fig. 3. The difference in intensity between the most and least intense regions is a factor 3.1. In contrast to the standard background (left 
Table 2. LECS observations used to create the standard background spectrum. A target name including "sec" or "secondary" refers to the prime WFC target. $N$ is the number of individual pointings. $N_{\text {gal }}$ is the line of sight absorption in units of $10^{20}$ atom $\mathrm{cm}^{-2}$ (Dickey \& Lockman 1990)

\begin{tabular}{|c|c|c|c|c|c|c|c|c|c|}
\hline \multirow[t]{2}{*}{ Target } & \multicolumn{2}{|c|}{ Observation } & \multirow[t]{2}{*}{$N$} & \multicolumn{2}{|c|}{ Position (J2000) } & \multirow{2}{*}{$\begin{array}{r}l_{\mathrm{II}} \\
\left(^{\circ}\right)\end{array}$} & \multirow{2}{*}{$\begin{array}{l}b_{\text {II }} \\
\left({ }^{\circ}\right)\end{array}$} & \multirow{2}{*}{$\begin{array}{r}\text { Exp. } \\
(\mathrm{ks})\end{array}$} & \multirow[t]{2}{*}{$N_{\text {gal }}$} \\
\hline & $\begin{array}{c}\text { Start } \\
\text { (yr mn day) }\end{array}$ & $\begin{array}{c}\text { Stop } \\
\text { (yr mn day) }\end{array}$ & & $\begin{array}{c}\mathrm{RA} \\
(\mathrm{h} \mathrm{m} \mathrm{s})\end{array}$ & $\begin{array}{c}\text { Dec } \\
\left(\begin{array}{lll}0 & \prime & \prime \prime\end{array}\right)\end{array}$ & & & & \\
\hline Polaris Region & 1996 Jul. 01 & 1997 May 23 & 6 & 023142.0 & +891547 & 123.3 & +26.5 & 69.7 & 7.0 \\
\hline Gal cent-2 (sec) & 1996 Aug. 23 & 1996 Aug. 27 & 1 & 165119.5 & +601149 & 89.8 & +38.1 & 82.3 & 2.1 \\
\hline Gal cent-3 (sec) & 1996 Aug. 27 & 1996 Aug. 31 & 1 & 163510.7 & +594630 & 89.9 & +40.2 & 79.9 & 2.0 \\
\hline SGR-A (sec) & 1996 Oct. 10 & 1996 Oct. 12 & 1 & $\begin{array}{lll}06 & 12 & 22.7\end{array}$ & -605904 & 270.1 & -28.2 & 43.3 & 4.2 \\
\hline Secondary Target (S1) & 1997 Mar. 18 & 1997 Mar. 25 & 1 & 175646.1 & +611145 & 90.2 & +30.1 & 94.2 & 3.4 \\
\hline Secondary Target (S2) & 1997 Apr. 13 & 1997 Apr. 15 & 2 & 181820.5 & +605842 & 90.2 & +27.4 & 26.6 & 3.8 \\
\hline SDC Target & 1997 Dec. 13 & 1997 Dec. 14 & 1 & 230753.5 & +085006 & 84.4 & -46.1 & 13.9 & 4.7 \\
\hline Secondary Target (S4) & 1998 Mar. 10 & 1998 Mar. 22 & 2 & 055207.9 & -610535 & 270.0 & -30.6 & 46.7 & 5.3 \\
\hline Secondary Target (S5) & 1998 Aug. 22 & 1998 Oct. 01 & 4 & 175207.4 & +610101 & 89.9 & +30.6 & 102.2 & 3.5 \\
\hline
\end{tabular}

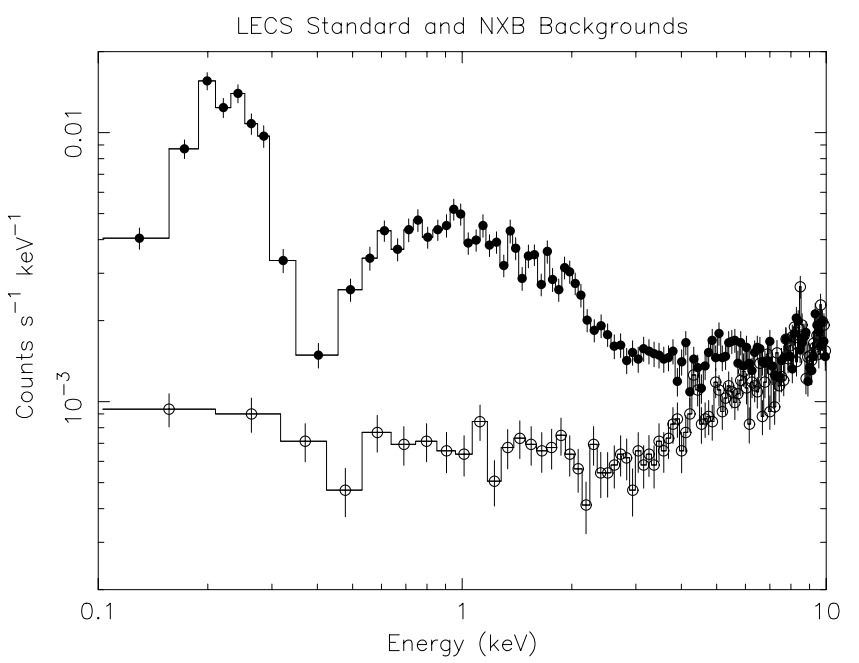

Fig. 4. Rebinned spectrum of the standard background (filled circles) and the NXB (open circles) in the central $8^{\prime}$ radius of the FOV

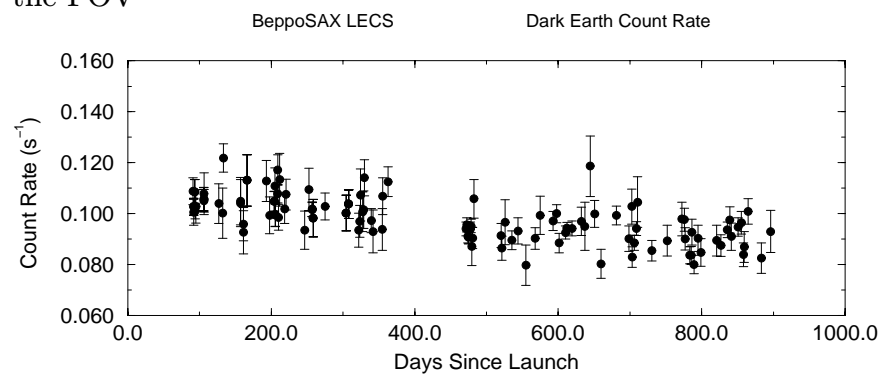

Fig. 5. The dependence of the FOV averaged LECS dark Earth count rate on time. The launch date was 1996 April 30

panel of Fig. 3), the NXB intensity near the center of the FOV does not show an enhancement. Within the central $5^{\prime}$ radius, any such increase is $<4 \%$ of the total intensity.

Spectra of the LECS NXB are shown in Figs. 4 and 6 . The overall level is approximately constant with energy with 3 discrete features superposed on a smooth increase $\gtrsim 4 \mathrm{keV}$. These features can be modeled as narrow Gaussian emission lines at $5.12 \pm 0.04 \mathrm{keV}$ (at $68 \%$ confi- dence), $8.51 \pm 0.04 \mathrm{keV}$ and $10.73 \pm 0.07 \mathrm{keV}$. The first feature shows a clear position dependence, being stronger close to the calibration sources. It is almost certainly produced by $5.9 \mathrm{keV}$ characteristic X-rays from the ${ }^{55} \mathrm{Fe}$ calibration sources that penetrate deeply into the detector before being absorbed. The detected energy is lower than the natural energy of the events, because X-rays absorbed deep within the detector produce, on average, less light than X-rays of the same energy absorbed close to the entrance window due to the different scintillation lengths in the driftless gas cell (Parmar et al. 1997b). The other 2 features do not exhibit an obvious position dependence within the FOV and may originate from fluorescent excitation of L-shell transitions in the tungsten window support structure (see Parmar et al. 1997b).

To illustrate the spectral changes associated with the decrease in count rate shown in Fig. 5, Fig. 6 shows the spectrum of the NXB before and after day 400. This shows that at energies $\lesssim 8 \mathrm{keV}$ the long term temporal evolution of the LECS NXB is not strongly energy dependent and may be simply modeled as the change in overall normalization given above. Above $8 \mathrm{keV}$, the intensity variation with time is less marked. This may indicate that the fluorescent line features exhibit less intensity variability than the rest of the spectrum.

The geomagnetic rigidity is a measure of the minimum momentum required by a cosmic particle to penetrate the Earth's magnetic field down to the position of the satellite. Due to the almost circular, low inclination BeppoSAX orbit, the variation in rigidity around the BeppoSAX orbit is less than is typical for low-Earth orbiting spacecraft such as ASCA which has an orbital inclination of $31^{\circ}$ and experiences rigidities between $6-14 \mathrm{GeV} \mathrm{c}^{-1}$. In the case of BeppoSAX, the rigidity typically varies from 10 to $16 \mathrm{GeV} \mathrm{c}^{-1}$ around the orbit. In order to investigate the dependence of the NXB spectrum on rigidity, spectra were accumulated for intervals when the rigidity was $\leq$ and $>13 \mathrm{GeV} c^{-1}$. The two spectra have almost 


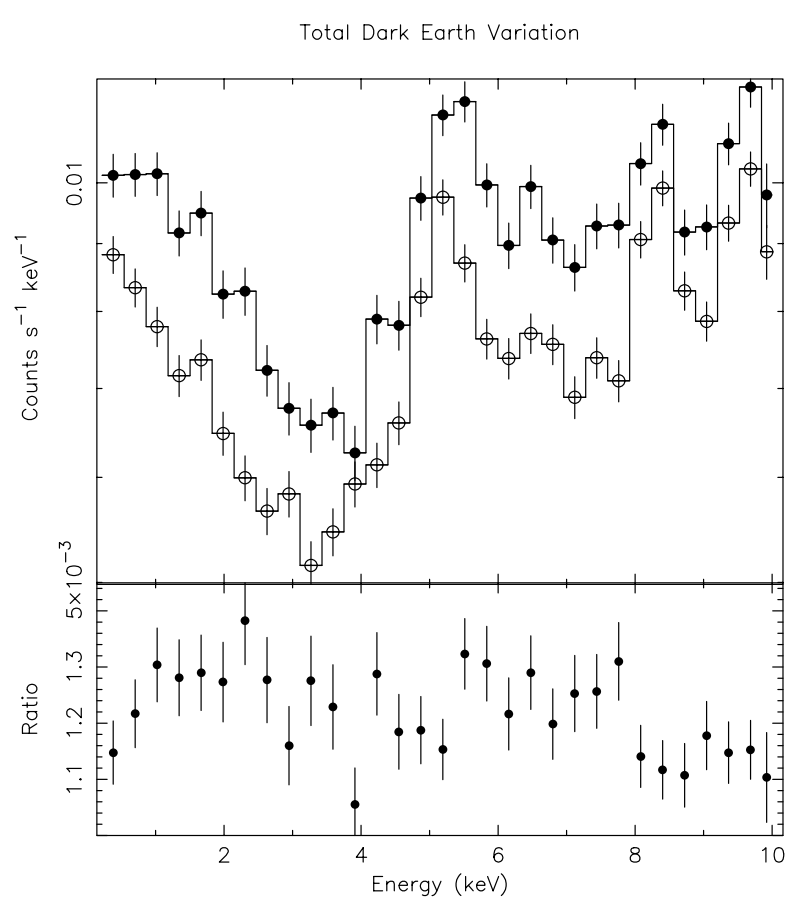

Fig. 6. The dependence of the rebinned LECS NXB spectrum, averaged over the entire FOV, on time. The filled circles show the spectrum obtained before day 400 and the open circles after day 400. The lower panel shows the ratio of the two spectra

identical shapes, with the spectrum accumulated when the rigidity was $\leq 13 \mathrm{GeV} \mathrm{c} c^{-1}$ having an overall normalization $5 \pm 1 \%$ higher. This means that for all but the shortest observations temporal averaging will ensure that the dependence of the NXB on geomagnetic rigidity can be ignored.

\section{Background semi-annuli}

The same data as in Sect. 5 were used to create a set of spectra extracted from within two semi-annuli located $17^{\prime}$ from the center of the FOV at positions diametrically opposite to the locations of the radioactive calibration sources (see Fig. 1). The location and sizes of the semi-annuli were carefully chosen to ensure that source "spill-over" is only significant for bright sources, while the correction for mirror vignetting is kept as small as possible. The sum of the semi-annuli geometric areas is equal to that of the standard $8^{\prime}$ radius source extraction region. Counts within the semi-annuli consist of both particle and internal background events (i.e., the NXB), sky background events, and any contribution from source photons that "spill-over" into the semi-annuli. The effects of "spill-over" are illustrated in Fig. 7 where the count rate in the semi-annuli, $C_{\text {ann }}$, is plotted against source count rate in the central $8^{\prime}$ radius source extraction region, $C_{\mathrm{src}}$. Extended sources such as supernova remnants and clusters of galaxies, observations where the target source is offset in the FOV, and pointings near the galactic center

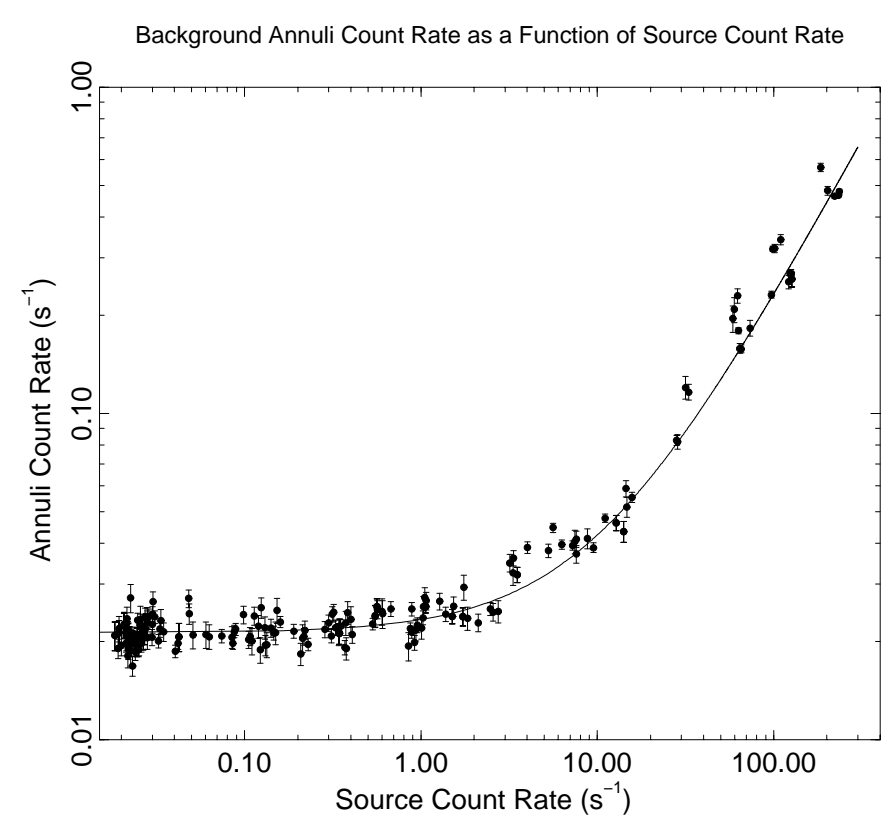

Fig. 7. The dependence of the $0.1-10 \mathrm{keV}$ background semiannuli count rate on the source count rate in the central $8^{\prime}$ radius extraction region. The solid line shows the best-fit model discussed in the text

Table 3. Coefficients of the fifth-order polynomial fit to the ratio of additional counts in the background semi-annuli divided by source counts as a function of energy in $\mathrm{keV}$. These coefficients can be used to correct counts extracted from the background semi-annuli for source contamination. $a_{0}$ is the constant term

\begin{tabular}{|c|c|c|c|}
\hline$a_{n}$ & Value & $a_{n}$ & Value \\
\hline$a_{0}$ & $-2.7110^{-3}$ & $a_{3}$ & $-6.1310^{-5}$ \\
\hline$a_{1}$ & $+4.1110^{-3}$ & $a_{4}$ & $-1.0110^{-5}$ \\
\hline$a_{2}$ & $+9.7910^{-4}$ & $a_{5}$ & $+9.5710^{-7}$ \\
\hline
\end{tabular}

are excluded. The solid line shows the fit to the expected linear relation: $C_{\mathrm{ann}}=0.0212+0.00212 C_{\mathrm{src}}$. This simple relation ignores the the energy dependence of "spill-over". It indicates that "spill-over" adds $<10 \%$ to the semi-annuli background for on-axis source count rates $<1 \mathrm{~s}^{-1}$.

The energy dependence of "spill-over" was investigated by subtracting the count rates obtained with blank fields from each of the semi-annuli spectra and dividing the remaining counts by those of the source with the standard background subtracted. Figure 8 shows a polynomial fit (Table 3) to the ratio derived in this way as a function of energy. There are at least two effects which produce these additional counts in the background semi-annuli. At lowenergies "spill-over" is dominated by the poor detector spatial resolution $\left(\propto\right.$ Energy $\left.^{-0.5}\right)$, while at high-energies the mirror scattering wings dominate.

The blank field exposures listed in Table 2 were also used to derive the offset correction factors which are shown in Fig. 9. Above $4 \mathrm{keV}$, where there are few sky background counts, the correction factors were derived 


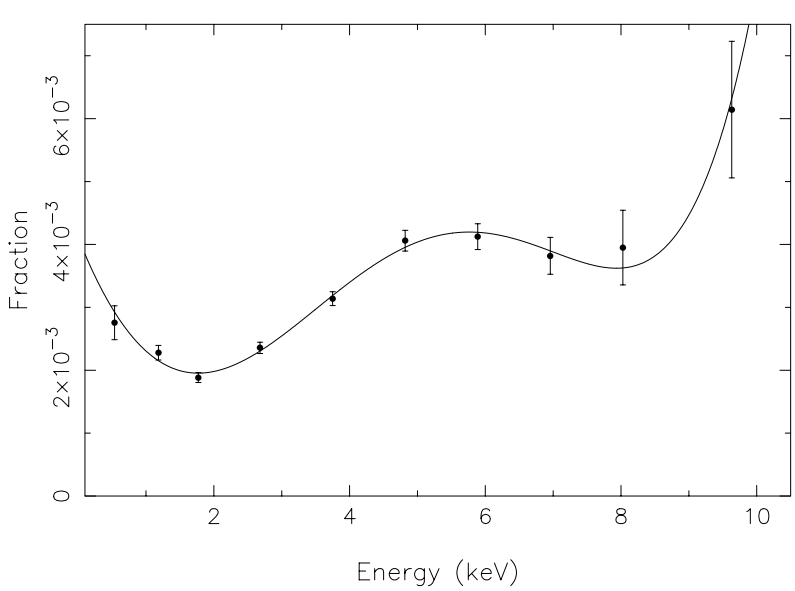

Fig. 8. Ratio of additional counts in the background semi-annuli divided by source counts as a function of energy. The solid line shows the fifth-order polynomial fit given in Table 3

by extrapolating results obtained from a Crab Nebula observation at an offset of $11^{\prime}$. Multiplication of the NXB subtracted spectrum in the semi-annuli by these factors gives the predicted background spectrum in the central $8^{\prime}$ radius source extraction region when the NXB is added. These correction factors are not simply the mirror vignetting function given in Conti et al. (1994) since, other factors such as an increase in PMT noise due to the lower signal levels off-axis and the position dependency of singularly reflected X-rays may contribute. The correction factors do not change significantly within the range of nominal target locations within the FOV of $\sim 2^{\prime}$ (see Sect. 3). Correction factors have also been determined for extraction radii of $4^{\prime}$ and $6^{\prime}$. These may be obtained by scaling the values in Fig. 9, which are for an extraction radius of $8^{\prime}$, by the ratio of extraction region areas. The ability to accurately derive these correction factors is limited by the low number of counts in the standard background exposure. As more LECS background fields become available, these correction factors will be updated.

\section{Background subtraction techniques}

In order to study the X-ray emission from a particular source it is usually necessary to subtract the X-ray and NXB contributions from within the extraction region. In the case of the LECS (and MECS) it is important to realize that strong sources outside the FOV (and within $2^{\circ}$ from the instrument axis) can contribute to the background. A search should be made for the presence of such sources using e.g., the HEASARC's XCOLL catalog.

In the case of the LECS, we present 3 ways of subtracting the background. An estimate of the systematic uncertainty associated with background subtraction may be obtained by comparing results obtained using the different methods.

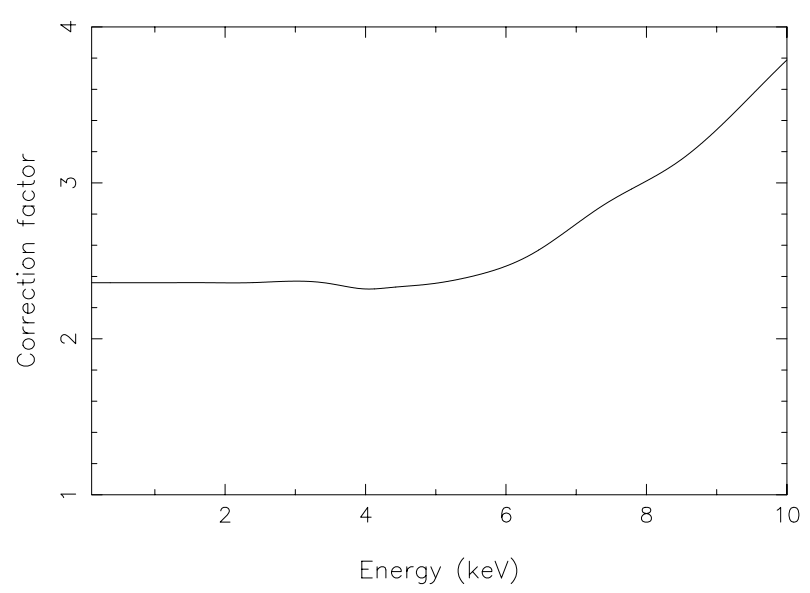

Fig. 9. Correction factors to be applied to a sky spectrum obtained from the background semi-annuli to give the predicted sky background in the standard $8^{\prime}$ radius source extraction region

\subsection{Standard background}

This is the simplest approach since the standard background includes the contribution from the high $\left(>|25|^{\circ}\right)$ galactic latitude CXB and the NXB. Since the standard background has a longer accumulation time (558.6 ks) than individual BeppoSAX observations, this method does not usually add significant noise. Since the standard background varies with position within the FOV (Fig. 3), the background spectrum should be accumulated using the same extraction region as the source. Note that this technique will underestimate the low-energy background for pointings in directions with bright Galactic emission such as the North Polar Spur (see Sect. 4).

\subsection{Background semi-annuli}

This technique has the following steps:

1. Accumulate a spectrum of the two background semiannuli during the observation of interest. These can be selected in RAWX, RAWY using the data extraction program XSELECT as follows:

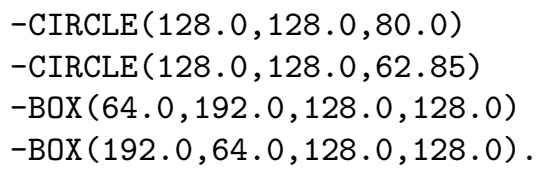

2. For source count rates $\gtrsim 1 \mathrm{~s}^{-1}$, subtract the NXB background in the standard extraction region from the total counts in the same region. Then multiply the subtracted counts by the function shown in Fig. 8 to derive the "spill-over" contribution. This is then subtracted from the extracted semi-annuli spectrum.

3. Subtract the standard NXB spectrum of the semiannuli.

4. Multiply the remaining spectrum by the correction factors shown in Fig. 9. This provides an estimate of the $\mathrm{CXB}$ in the source region. 
5. Add the standard NXB spectrum in the source region to this spectrum. This is then the final background to be subtracted before scientific analysis of the source properties.

\subsection{Scaled ROSAT PSPC all-sky survey background}

ROSAT PSPC all-sky survey diffuse background maps are available in 7 energy bands with a pixel size of $12^{\prime} \times 12^{\prime}$ covering $\sim 98 \%$ of the sky. The effects of discrete source counts, non X-ray contamination and X-rays of solar system origin have been eliminated to the greatest possible extent. The contributions of all the sources listed in Table 4 have been excluded. These maps can be used to predict the variation with pointing position of the LECS background. The PSPC count rates closest to the centers of each of the fields included in the LECS standard background were first determined. The mean, LECS exposure weighted, PSPC $0.1-2.5 \mathrm{keV}$ count rate is $1.210^{-3} \mathrm{~s}^{-1} \operatorname{arcmin}^{-2}$.

In order to understand how best to model the PSPC data from different sky positions, 7 channel PSPC spectra were determined for the fields given in Tables 2 and 4 . These were then fit with a number of different models including power-laws, thermal bremsstrahlungs, broken power-laws with the hard component $\alpha$ fixed at 1.4 (the value expected above $1 \mathrm{keV}$ ), and power-laws with $\alpha=1.4$, together with thermal components. In all cases low-energy absorption was included. It was found that (1) the power-law and (2) the fixed power-law together with a thermal component give consistently the best fits for almost all the fields. These two models are therefore used in the background analysis except for the Gal cent-3 field, where a broken power-law, with hard index $\alpha$ fixed at 1.4 is preferred.

In order to use this technique to predict the LECS background spectrum at a particular pointing position, the following steps are necessary:

1. Determine the ROSAT PSPC count rates in the 7 energy bands at the required position (see Sect. 9).

2. Fit spectral models to the derived spectrum using the appropriate ROSAT all-sky survey response matrix.

3. Select the model with the lowest reduced $\chi^{2}$.

4. Use the standard LECS response matrix and the parameters of the selected model fit to calculate a predicted background spectrum for the position of interest.

5. Use the standard LECS response matrix and the CXB fit parameters given below to calculate a predicted spectrum for the standard background field.

6. Divide the predicted LECS spectrum by that predicted for the standard background to derive a set of PI channel dependent correction factors.
7. Subtract the standard NXB spectrum (see Sect. 5) measured in the source extraction region from the $o b$ served standard background spectrum.

8. Multiply the subtracted spectrum by the correction factors calculated above.

9. Add the standard NXB measured in the source extraction region from the standard background spectrum to the scaled spectrum. This is then the final background to be subtracted before scientific analysis of the source properties.

The LECS CXB spectrum is discussed in a companion $p a-$ per (Parmar et al 1999). In the $0.1-7.0 \mathrm{keV}$ energy range it is well modeled by an absorbed power-law together with two solar abundance thermal bremsstrahlung components (the MEKAL model in XSPEC). When this model is fit to the exposure weighted PSPC spectrum corresponding to the standard background, the best-fit results are a power-law $\alpha$ of 1.48 with normalization of $1.0710^{-3}$ photon $\mathrm{cm}^{-2} \mathrm{~s}^{-1} \mathrm{keV}^{-1}$, a hard bremsstrahlung component temperature $(k T)$ of $0.80 \mathrm{keV}$ with normalization of $1.510^{-5}$ photon $\mathrm{cm}^{-5}$, and a soft bremsstrahlung component $k T$ of $0.159 \mathrm{keV}$ with normalization of $1.910^{-4}$ photon $\mathrm{cm}^{-5}$. For both absorbed models, $N_{\mathrm{H}}$ equivalent to $3.710^{20}$ atom $\mathrm{cm}^{-2}$ is required. These fit parameters should be used to generate the predicted standard LECS background spectrum.

\section{Background subtraction comparison}

The accuracy of the different background subtraction techniques described in Sect. 7 was investigated using both blank field and exposures containing sources. The blank field exposures are listed in Table 2 and the source exposures in Table 4.

\subsection{Target selection}

CAL 83 and CAL 87 are two "supersoft" sources located in the Large Magellanic Cloud. CAL 83 is only detected in the LECS between $0.1-0.6 \mathrm{keV}$ (Parmar et al. 1998) and CAL 87 between $0.2-1.0 \mathrm{keV}$ (Parmar et al. 1997a). HZ 43 is a nearby hot white dwarf (e.g., Barstow et al. $1995)$ that is often used as an extreme ultra violet or soft X-ray "standard candle" in X-ray astronomy. Due to the softness of its spectrum, it is only detected below $0.28 \mathrm{keV}$ in the LECS. These 3 targets were chosen since they are only detected in a narrow energy range of the LECS, allowing the rest of the spectrum to be used to estimate the background subtraction quality.

$4 \mathrm{U} 1630-47$ is a recurrent X-ray transient located close to the galactic plane. The BeppoSAX observation was designed to detect quiescent emission between outbursts, but none was found (Oosterbroek et al. 1998). X 1755-338 is a bright X-ray dipping source which was observed to have 

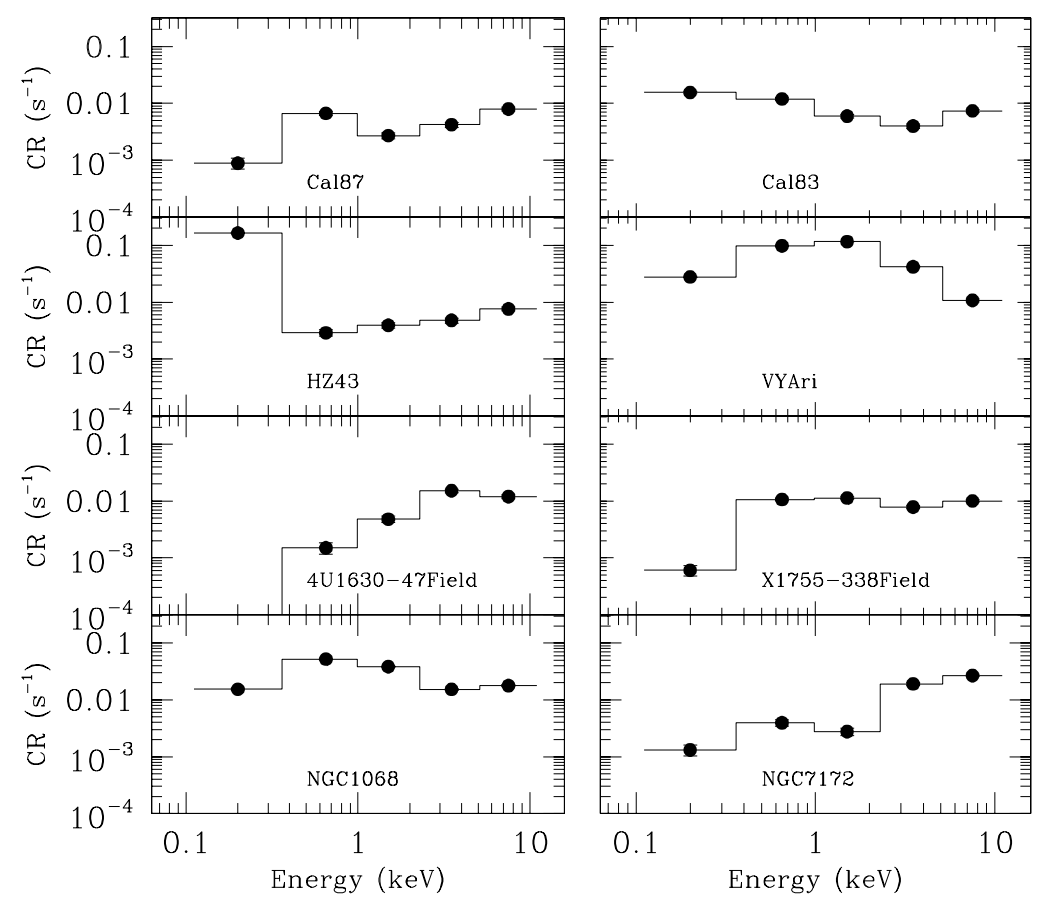

Fig. 10. Count rates (CR) for the source sample in the energy ranges $0.1-0.3,0.3-1,1-2,2-5$, and $5-10 \mathrm{keV}$. The counts include contributions from the source (if present) and any extended emission components within the $8^{\prime}$ extraction radii. The ordinate uncertainties are smaller than the symbol size
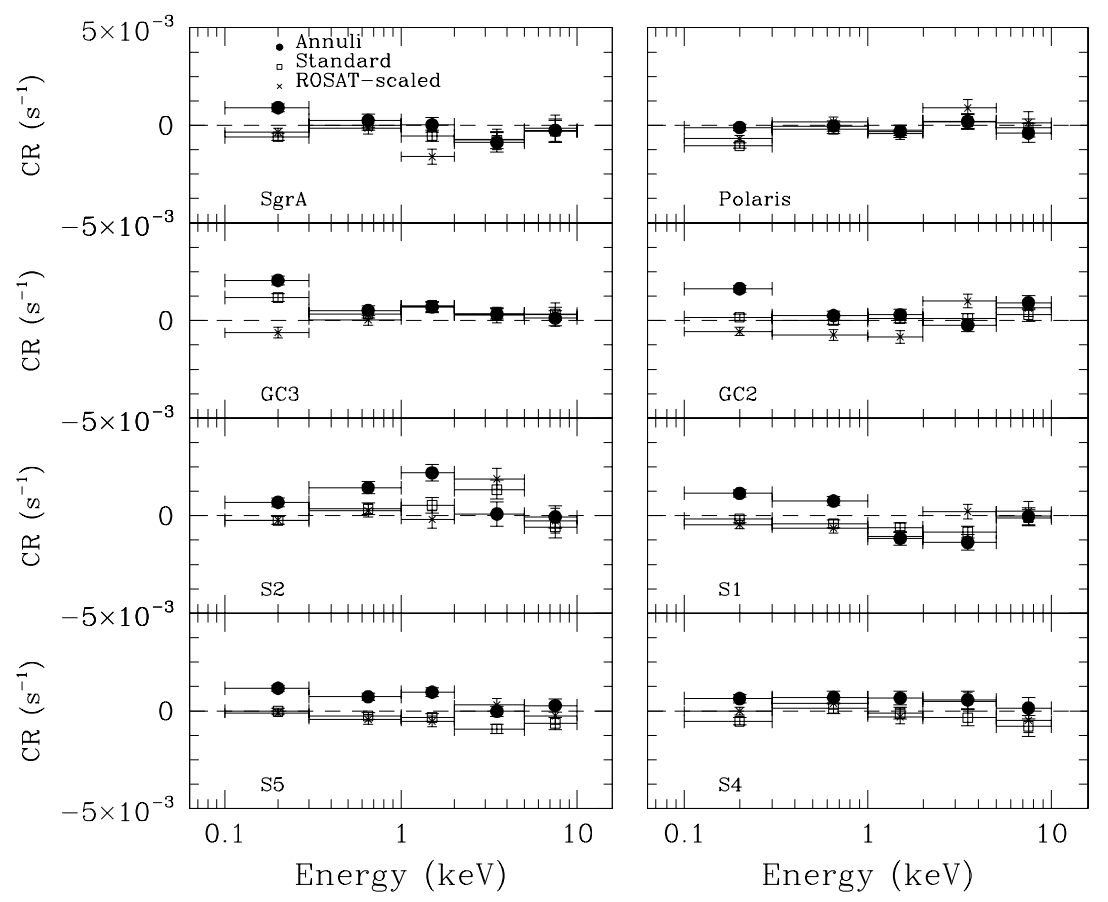

Fig. 11. Count rate residuals (CR) as a function of energy for 8 blank fields from Table 2, when the 3 background subtracted techniques are applied. Empty squares indicate the standard background, filled circles the semi-annuli, and crosses the ROSATscaled techniques 


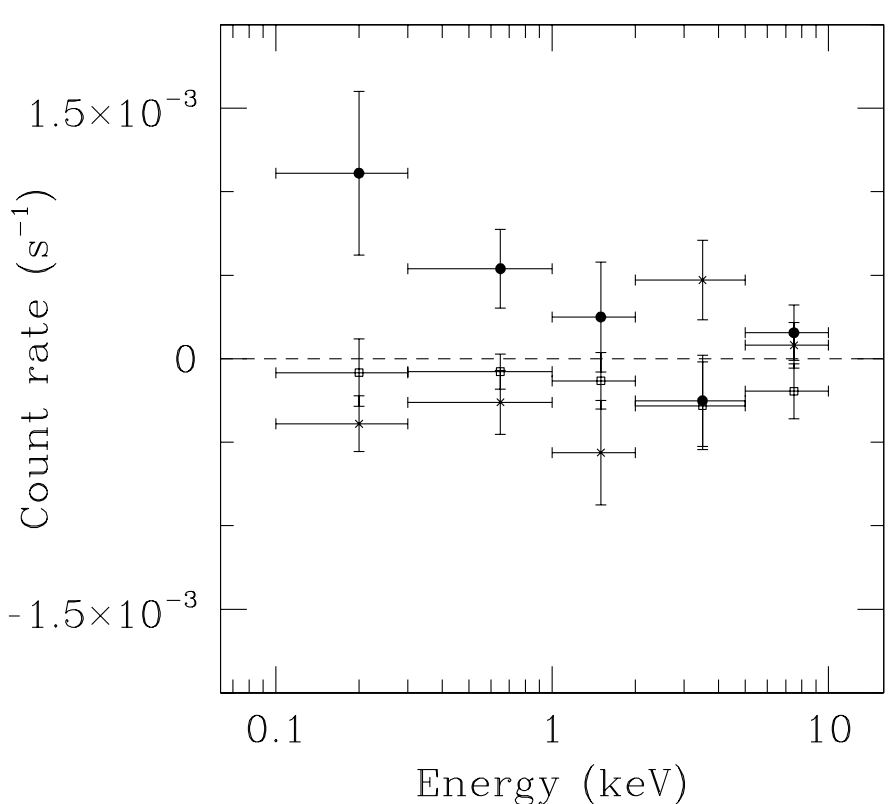

Fig. 12. Exposure time weighted sum of the 8 blank field exposure residuals when the 3 background subtraction techniques are applied. Empty squares indicate the standard background, filled circles the semi-annuli, and crosses the ROSAT-scaled techniques

turned-off prior to the BeppoSAX observation (Roberts et al. 1996). These two fields were chosen as tests of the background subtraction techniques in complex regions of the sky at low galactic latitudes.

NGC 7172 is a Seyfert 2 galaxy, whose nuclear continuum is seen through an $N_{\mathrm{H}} \sim 10^{23}$ atom $\mathrm{cm}^{-2}$ neutral absorber. A soft excess observed by ASCA was associated with diffuse emission from the group HGC 90, in which NGC 7172 is located (Guainazzi et al. 1998). In addition, two relatively bright $\left(\sim 0.25\right.$ count $\left.\mathrm{s}^{-1}\right)$ sources are included as a control sample, to show the relative independence of the results on the choice of the background subtraction method at higher flux levels. These are the Seyfert 2 Galaxy NGC 1068 (Guainazzi et al. 1999) and the coronal X-ray source VY Ari (Favata et al. 1997).

\subsection{Results}

In Fig. 10, observed LECS count rates in five energy bands $(0.1-0.3,0.3-1,1-2,2-5$, and $5-10 \mathrm{keV})$ are shown for the sample sources. No correction for the instrumental response has been applied. All results in this section are displayed in this way to allow ease of comparison since count rate is a quantity which can be easily estimated when comparing the behavior of a weak X-ray source with the instrumental performances and limitations.

Figure 11 shows the residuals for 8 blank fields when each of the 3 background subtraction techniques are applied. (The shortest exposure blank field, the SDC Target, in Table 2 was excluded.) In the case of a good background
Table 5. The values of $|\mu|+3 \sigma$ for the 3 background subtraction techniques in 3 energy ranges for the blank fields in units of $10^{-3}$ count s ${ }^{-1}$

\begin{tabular}{lccc}
\hline Method & $0.1-10 \mathrm{keV}$ & $0.1-2 \mathrm{keV}$ & $2-10 \mathrm{keV}$ \\
\hline Standard & 0.8 & 0.7 & 1.3 \\
Semi-annuli & 1.4 & 1.9 & 1.2 \\
ROSAT-scaled & 0.9 & 1.3 & 1.3 \\
\hline
\end{tabular}

subtraction, the residuals should exhibit a Poissonian distribution centered on zero, and the standard deviation of the residuals provides an estimate of the systematic uncertainties associated with each technique. These results are summarized in Fig. 12, where the exposure time weighted sum of the background field residuals, shown individually in Fig. 11, are plotted. The average mean count rates, $\mu$, in the $0.1-10 \mathrm{keV}$ energy range are $(-1.5 \pm 0.9),(3.6 \pm 1.6)$ and $(-1.3 \pm 1.0) 10^{-4} \mathrm{~s}^{-1}$, for the standard, semi-annuli and ROSAT-scaled technique, respectively. Table 5 lists the values of $|\mu|+3 \sigma$ ( $\sigma$ is the standard deviation) for the 3 techniques in 3 energy ranges. These values may be interpreted as $3 \sigma$ estimates of the systematic uncertainties of each the background subtraction techniques at high galactic latitudes. In the $0.1-2 \mathrm{keV}$ energy range, the values in Table 5 correspond to fluxes in the range $0.7-1.8$ and $0.5-1.110^{-13} \mathrm{erg} \mathrm{cm}^{-2} \mathrm{~s}^{-1}$ for a power-law spectrum with $\alpha=2.0$ and photoelectric absorption, $N_{\mathrm{H}}$, of $310^{20}$ and $310^{21}$ atom $\mathrm{cm}^{-2}$, respectively. In the $2-10 \mathrm{keV}$ band, for an $N_{\mathrm{H}}$ of $310^{20}$ atom $\mathrm{cm}^{-2}$, the limiting fluxes are $2-3$ and $1.3-310^{-13} \mathrm{erg} \mathrm{cm}^{-2} \mathrm{~s}^{-1}$ for sources with $\alpha=2.0$ and 1.5 , respectively.

In the case of the blank fields, the standard background provides the most consistent (i.e., lowest values of $|\mu|+3 \sigma)$ subtraction. This is unsurprising given that the standard background itself includes the comparison fields (see Table 2). In addition, the blank field exposures are at high $\left(>|25|^{\circ}\right)$ galactic latitude and avoid features such as the North Polar Spur, and so are expected to be broadly similar. The residuals obtained with the semi-annuli method are the largest. An investigation reveals that this may be in part due to uncertainties in the NXB spectra of the two semi-annuli which contain $<8800$ counts. It is expected that this will improve as subsequent dark Earth exposures are added to the already existing data set. The ROSAT-scaled background works consistently well within the limits specified in Table 5.

Figure 13 illustrates the application of the 3 background subtracted techniques to the sample sources. For each source the 3 techniques were applied and background subtracted count rates in the 5 energy bands calculated. The difference with respect to the mean of the 3 techniques is plotted in Fig. 13. In 6 out of 8 cases no significant deviations at levels higher than a few $\times 10^{-3}$ count $\mathrm{s}^{-1}$ are present. This is comparable to the results on the blank sky fields and indicates that all three methods work well 
Table 4. Observations used for background accuracy checks. CR is LECS count rate including the contribution of the background in the $8^{\prime}$ radius extraction regions

\begin{tabular}{llcrrl}
\hline $\begin{array}{l}\text { Source } \\
\text { Name }\end{array}$ & $\begin{array}{l}\text { CR } \\
\left(\mathrm{s}^{-1}\right)\end{array}$ & $\begin{array}{c}\text { Date } \\
(\text { yr mn day })\end{array}$ & \multicolumn{1}{c}{$\begin{array}{c}l_{\text {II }} \\
\left(^{\circ}\right)\end{array}$} & \multicolumn{1}{c}{\begin{tabular}{c}
\multicolumn{1}{c}{$\left(_{\text {II }}\right.$} \\
( $)$
\end{tabular}} & Remarks \\
\hline CAL 87 & 0.031 & 1996 Oct. 27 & 281.8 & -30.7 & Supersoft source \\
4U 1630-47 Field & 0.040 & 1997 Mar. 26 & 336.9 & +0.3 & X-ray transient in quiescence \\
CAL 83 & 0.043 & 1997 Mar. 07 & 278.6 & -31.3 & Supersoft source \\
X 1755-338 Field & 0.045 & 1998 Apr. 10 & 357.2 & -4.9 & X-ray binary in quiescence \\
NGC 7172 & 0.059 & 1996 Oct. 14 & 321.2 & -18.6 & Active Galactic Nuclei \\
NGC 1068 & 0.142 & 1996 Dec. 30 & 172.1 & -51.9 & Active Galactic Nuclei \\
HZ 43 & 0.215 & 1998 Jan. 27 & 54.1 & +84.2 & Hot white dwarf \\
VY Ari & 0.303 & 1996 Sep. 06 & 150.6 & -25.4 & Coronal X-ray emission \\
\hline
\end{tabular}
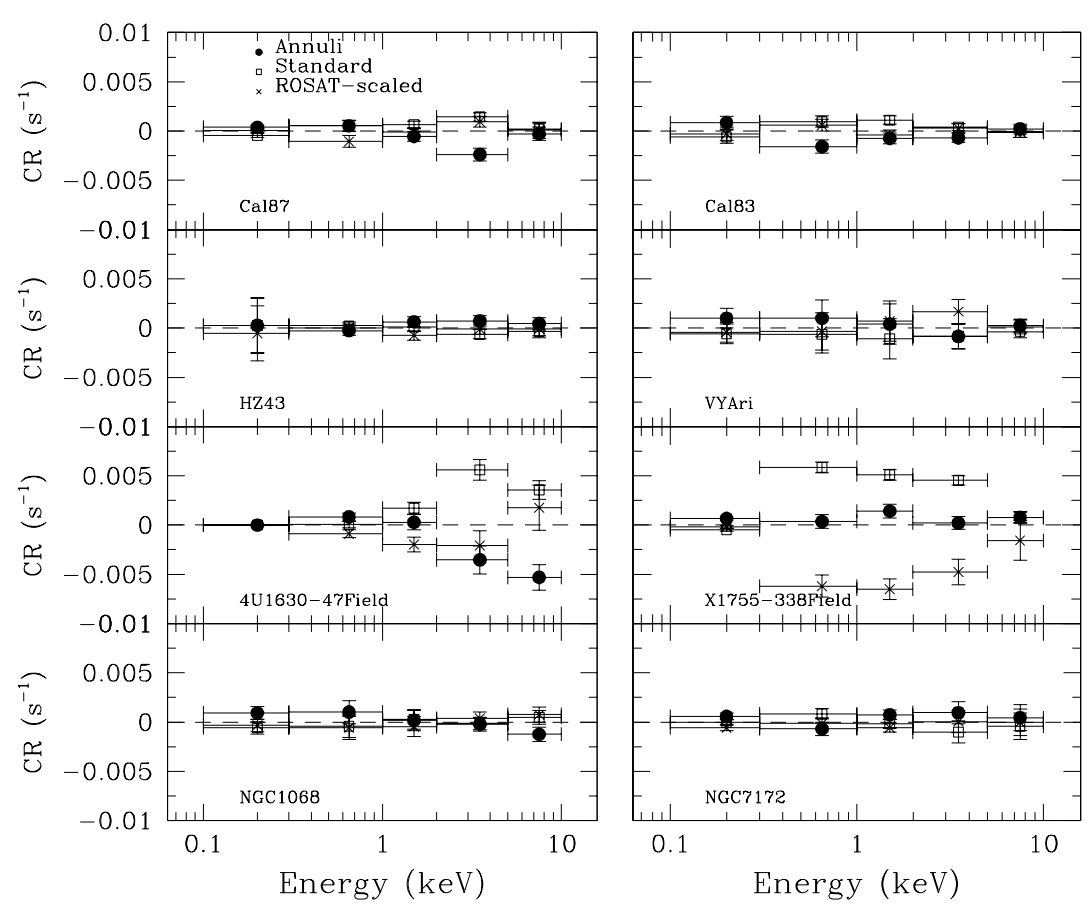

Fig. 13. Count rate difference compared to the mean $(\mathrm{CR})$ as a function of energy when the 3 background subtraction techniques are applied to each of the sample sources. Empty squares indicate the standard background technique, filled circles the semi-annuli, and crosses the ROSAT-scaled technique. Note that the ordinate extrema are a factor 2 larger than in Fig. 11

here. The differences between the three methods may be used to estimate the systematic uncertainties associated with background subtraction.

In the case of the two fields close to the galactic plane (4U 1630-47 and X 1755-338), differences at a level $\lesssim 10^{-3}$ count $\mathrm{s}^{-1}$ are evident. This is a factor $\sim 2.5$ larger than with the blank field exposures and is probably due to incorrect estimation of the contributions of the hard diffuse emission associated with the Galactic ridge, or unresolved point-sources. In addition, in the case of $4 \mathrm{U} 1630-47$ the $50 \mathrm{mCrab}$ (or $\sim 10$ LECS count $\mathrm{s}^{-1}$ ) $\mathrm{X}$-ray binary $4 \mathrm{U} 1624-49$ is located $2^{\circ}$ away. X-rays from $4 \mathrm{U} 1624-49$ that undergo a single mirror reflection may provide a small contribution to the $4 \mathrm{U} 1630-47$ field.

In both these cases, the standard background systematically underestimates the background (as expected), and the scaled ROSAT method overestimates the background somewhat, when compared to the mean values. The reason for this is unclear, but it may be partly due to incorrect ROSAT source subtraction in the complex fields. In the case of the X 1755-338 field, the residuals obtained using the semi-annuli method are smoothly distributed between those obtained using the other two methods. This is not the case in the 4U1630-47 field where the residuals deviate from the mean values above $2 \mathrm{keV}$. These results suggest that the semi-annuli gives the most reliable results for complex fields, with the scaled ROSAT method being the second most reliable. These two cases illustrate the difficulty in obtaining a good background subtraction for sources located at low galactic latitudes, or in complex regions of the X-ray sky. 
Finally, the effects of the long term decrease in NXB intensity evident in Fig. 5 were evaluated using all three proposed background subtraction techniques. In all cases the differences in background residuals were substantially smaller than the values given in Table 5. This means that currently no time dependent corrections need to be applied to the LECS background.

\section{Data products}

The standard background and NXB cleaned event lists will be made available by the BeppoSAX Science Data Center in Rome. These files will allow users to generate appropriate background and NXB spectra.

In addition, the Max-Planck-Institut für Extraterrestrische Physik, in Garching will make the 7 energy channel source subtracted PSPC maps available through the World Wide Web so that users can use the scaled ROSAT background subtraction technique.

Acknowledgements. The BeppoSAX satellite is a joint ItalianDutch programme. M. Guainazzi, T. Oosterbroek, and A. Orr acknowledge ESA Fellowships and N. Shane the ESA stagiaire programme. We thank F. Fiore for pointing out the importance of single reflection X-rays to this study, and S. Molendi and S. Snowden for helpful discussions. The support of M. Werger and O.R. Williams in maintaining the SSD BeppoSAX archive is appreciated. Finally, we thank all the BeppoSAX Principal Investigators who allowed their data to be used for this study prior to them becoming public.

\section{References}

Barstow M.A., Holberg J.B., Koester D., 1995, MNRAS 274, L31

Boella G., Butler R.C., Perola G.C., et al., 1997a, A\&AS 122, 299

Boella G., Chiappetti L., Conti G., et al., 1997b, A\&AS 122, 327
Chiappetti L., Cusumano G., Del Sordo S., et al., 1998, Nucl. Phys. B (Proc. Suppl.) 69/1-3, p. 610

Conti G., Mattaini E., Santambrogio E.B., et al., 1994, SPIE 2279,101

Conti G., Chiappetti L., Molendi S., et al., 1996, SPIE 3113, 394

Dickey J.M., Lockman F.J., 1990, ARA\&A 28, 215

Favata F., Mineo T., Parmar A.N., Cusumano G., 1997, A\&A 324,41

Frontera F., Costa E., Dal Fiume D., et al., 1997, A\&AS 122, 371

Guainazzi M., Matt G., Antonelli L.A., et al., 1998, MNRAS 298,824

Guainazzi M., Matt G., Antonelli L.A., et al., 1999 (in preparation)

Jager R., Mels W.A., Brinkmann A.C., et al., 1997, A\&AS 125, 557

Kerp J., 1994, A\&A 289, 597

Manzo G., Giarrusso S., Santangelo A., et al., 1997, A\&AS 122,341

Moritz P., Wennmacher A., Herbstmeier U., et al., 1998, A\&A 336,682

Oosterbroek T., Parmar A.N., Kuulkers E., et al., 1998, A\&A (in press)

Parmar A.N., Kahabka P., Hartmann H.W., et al., 1997a, A\&A 323, L33

Parmar A.N., Martin D.D.E., Bavdaz M., et al., 1997b, A\&AS 122,309

Parmar A.N., Kahabka P., Hartmann H.W., Heise J., Taylor B.G., 1998, A\&A 332, 199

Parmar A.N., Guainazzi M., Oosterbroek T., et al., 1999 (in preparation)

Roberts M.S.E., Michelson P.F., Cominsky L., et al., 1996, IAU Circ. 6302

Snowden S.L., Freyberg M.J., 1993, ApJ 404, 403

Snowden S.L., Freyberg M.J., Plucinsky P.P., et al., 1995, ApJ 454,643

Snowden S.L., Egger R., Freyberg M.J., et al., 1997, ApJ 485, 125

Snowden S.L., 1998, ApJS 117, 233

Voges W., Aschenbach B., Boller Th., et al., 1996, IAU Circ. 6420 